Online

ISSN 1600-5368
Acta Crystallographica Section E

Structure Reports

\section{2-Butyl-4-chloro-1-(4-nitrobenzyl)-1H- imidazole-5-carboxaldehyde}

The asymmetric unit of the title compound, $\mathrm{C}_{15} \mathrm{H}_{16} \mathrm{ClN}_{3} \mathrm{O}_{3}$, comprises two molecules that are each twisted about the benzyl $\mathrm{C}$ atom. The second and fourth $\mathrm{C}$ atoms of the butyl chain of one molecule are disordered (0.75:0.25 and 0.5:0.5, respectively). The dihedral angles between the imidazole and benzene rings are $76.46(9)$ and $76.3(1)^{\circ}$.

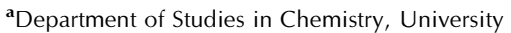
of Mysore, Manasagangotri, Mysore 570 006, India, and ${ }^{\mathbf{b}}$ School of Science and the Environment, Coventry University, Coventry CV1 5FB, England

Correspondence e-mail: apx106@coventry.ac.uk

Key indicators

Single-crystal X-ray study

$T=120 \mathrm{~K}$

Mean $\sigma(\mathrm{C}-\mathrm{C})=0.004 \AA$

Disorder in main residue

$R$ factor $=0.057$

$w R$ factor $=0.139$

Data-to-parameter ratio $=14.4$

For details of how these key indicators were automatically derived from the article, see http://journals.iucr.org/e.
(C) 2004 International Union of Crystallography Printed in Great Britain - all rights reserved

\section{Comment}

Imidazole derivatives are reported to be biologically active molecules, and both imidazoles and benzimidazoles are components of larger molecules used in pharmaceuticals, agrochemicals, dyestuffs and high-temperature polymer products (Rasmussen, 1999; Ambalvanan et al., 2003). With this in mind, the title compound, (I), was prepared in a series of syntheses to produce new imidazole derivatives. The Cambridge Structural Database (Version of April 2004; Allen, 2002) reveals that there are currently 42 known structures containing a 3-benzylimidazole moiety, but not yet the title compound.

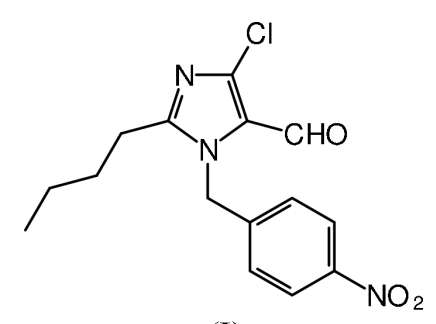

(I)

The asymmetric unit of (I) comprises two molecules, $A$ and $B$, that are each twisted about the benzyl $\mathrm{C}$ atom (Fig. 1). The butyl chain of molecule $B$ is disordered, with the second and fourth $\mathrm{C}$ atoms in the chain occupying two sites each. The second $\mathrm{C}$ atom is unequally disordered, with occupancies of 0.75:0.25 for $\mathrm{C} 22 B$ and $C 22 C$, respectively, whereas the fourth $\mathrm{C}$ atom is equally disordered across two sites (C24B and $\mathrm{C} 24 C)$. In early refinements, the third C atom (C23B) was split (similar to $\mathrm{C} 22 \mathrm{~B} / \mathrm{C}$ ), but this proved not to be a viable option, with the lesser refining unsatisfactorily. Stable refinement was achieved with $\mathrm{C} 23 B$ being treated as a whole atom, even though it displays larger displacement ellipsoids compared with neighbouring atoms. The dihedral angles between the imidazole and benzene rings are $76.46(9)$ and $76.3(1)^{\circ}$ for molecules $A$ and $B$, respectively.

\section{Experimental}

The title compound was prepared by stirring an equimolar mixture of 2-butyl-5-chloro-3H-imidazole-4-carboxaldehyde, 4-nitrobenzyl-
Received 25 November 2004 Accepted 26 November 2004 Online 30 November 2004 
bromide and $\mathrm{K}_{2} \mathrm{CO}_{3}$ in dimethylformamide at room temperature for $6 \mathrm{~h}$. The product was filtered and recrystallized from ethanol to yield colourless plates.

\section{Crystal data \\ $\mathrm{C}_{15} \mathrm{H}_{16} \mathrm{ClN}_{3} \mathrm{O}_{3}$ \\ $M_{r}=321.76$ \\ Triclinic, $P \overline{1}$ \\ $a=8.3007(5) \AA$ \\ $b=12.2295(6) \AA$ \\ $c=16.5605(10) \AA$ \\ $\alpha=103.420(4)^{\circ}$ \\ $\beta=95.561(3)^{\circ}$ \\ $\gamma=106.758(3)^{\circ}$ \\ $V=1541.35(15) \AA^{3}$}

$$
\begin{aligned}
& Z=4 \\
& D_{x}=1.387 \mathrm{Mg} \mathrm{m}^{-3} \\
& \text { Mo } K \alpha \text { radiation } \\
& \text { Cell parameters from } 6811 \\
& \quad \text { reflections } \\
& \theta=2.9-27.5^{\circ} \\
& \mu=0.26 \mathrm{~mm}^{-1} \\
& T=120(2) \mathrm{K} \\
& \text { Plate, colourless } \\
& 0.36 \times 0.30 \times 0.04 \mathrm{~mm}
\end{aligned}
$$

\section{Data collection}

Bruker-Nonius KappaCCD areadetector diffractometer

$\varphi$ and $\omega$ scans

Absorption correction: multi-scan (SADABS; Sheldrick, 1997b)

$T_{\min }=0.911, T_{\max }=0.990$

30632 measured reflections

\section{Refinement}

Refinement on $F^{2}$

$R\left[F^{2}>2 \sigma\left(F^{2}\right)\right]=0.057$

$w R\left(F^{2}\right)=0.139$

$S=1.04$

6038 reflections

418 parameters

$\mathrm{H}$-atom parameters constrained

6038 independent reflections 3622 reflections with $I>2 \sigma(I)$ $R_{\text {int }}=0.098$

$\theta_{\text {max }}=26.0^{\circ}$

$h=-10 \rightarrow 10$

$k=-15 \rightarrow 15$

$l=-20 \rightarrow 20$

$$
\begin{gathered}
w=1 /\left[\sigma^{2}\left(F_{o}{ }^{2}\right)+(0.0645 P)^{2}\right. \\
+0.076 P] \\
\text { where } P=\left(F_{o}{ }^{2}+2 F_{c}^{2}\right) / 3 \\
(\Delta / \sigma)_{\max }<0.001 \\
\Delta \rho_{\max }=0.29 \AA^{\circ} \AA^{-3} \\
\Delta \rho_{\min }=-0.34 \mathrm{e}^{-3}
\end{gathered}
$$

All $\mathrm{H}$ atoms were included in the refinement at calculated positions in the riding-model approximation, with $\mathrm{C}-\mathrm{H}$ distances of 0.95 (aromatic $\mathrm{H}$ atoms and $\mathrm{CHO} \mathrm{H}$ atoms), $0.98\left(\mathrm{CH}_{3} \mathrm{H}\right.$ atoms) and $0.99 \AA\left(\mathrm{CH}_{2} \mathrm{H}\right.$ atoms $)$. The isotropic displacement parameters were set equal to $1.25 U_{\text {eq }}$ of the carrier atom. A high $R_{\text {int }}$ was the result of weak high-angle data.

Data collection: COLLECT (Hooft, 1998); cell refinement: DENZO (Otwinowski \& Minor, 1997) and COLLECT; data reduction: DENZO and COLLECT; program(s) used to solve structure: SHELXS97 (Sheldrick, 1997a); program(s) used to refine structure:

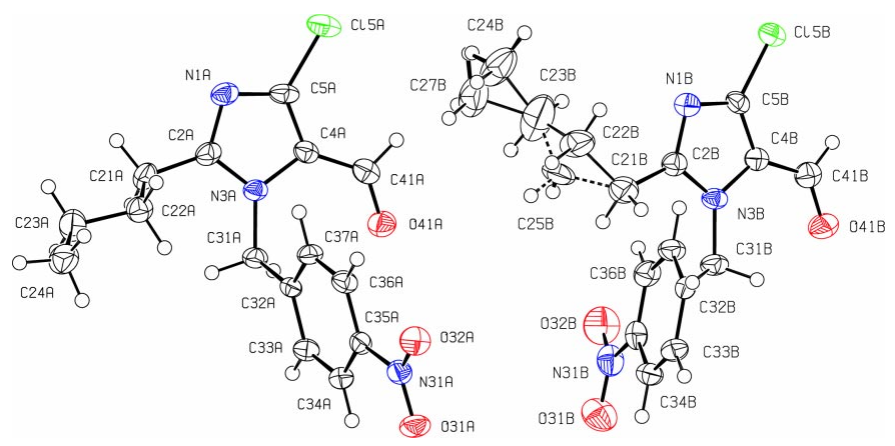

Figure 1

The molecular configurations and atom-numbering schemes for the two independent molecules, $A$ and $B$, of (I). Displacement ellipsoids are drawn at the $50 \%$ probability level and $\mathrm{H}$ atoms are shown as spheres of arbitrary radii.

SHELXL97 (Sheldrick, 1997a); molecular graphics: PLATON97 (Spek, 2003); software used to prepare material for publication: SHELXL97.

The authors thank the EPSRC National Crystallography Service (Southampton, England) and acknowledge the use of the EPSRC's Chemical Database Service at Daresbury.

\section{References}

Allen, F. H. (2002). Acta Cryst. B58, 380-388.

Ambalvanan, P., Palani, K., Ponnuswamy, M. N., Thirumuruhan, R. A., Yathirajan, H. S., Prabhuswamy, B., Raju, C. R., Nagaraja, P. \& Mohana, K. N. (2003). Mol. Cryst. Liq. Cryst. 393, 75-82.

Hooft, R. W. W. (1998). COLLECT. Nonius BV, Delft, The Netherlands.

Otwinowski, Z. \& Minor, W. (1997). Methods in Enzymology, Vol. 276, Macromolecular Crystallography, Part A, edited by C. W. Carter Jr \& R. M. Sweet, pp. 307-326. New York: Academic Press.

Rasmussen, P. G. (1999). J. Chem. Educ. 76, 1345.

Sheldrick, G. M. (1997a). SHELXS97 and SHELXL97. University of Göttingen, Germany.

Sheldrick, G. M. (1997b). SADABS. Version 2.10. Bruker AXS Inc., Madison, Wisconsin, USA.

Spek, A. L. (2003). J. Appl. Cryst. 36, 7-13. 


\section{supporting information}

Acta Cryst. (2004). E60, o2520-o2521 [https://doi.org/10.1107/S1600536804031162]

\section{2-Butyl-4-chloro-1-(4-nitrobenzyl)-1 H-imidazole-5-carboxaldehyde}

Santhosh L. Gaonkar, Hemmige S. Yathirajan, Basavegowda Nagaraj, Rajenahally S.

Narasegowda and Daniel E. Lynch

2-Butyl-4-chloro-1-(4-nitrobenzyl)-1 $H$-imidazole-5-carboxaldehyde

Crystal data

$\mathrm{C}_{15} \mathrm{H}_{16} \mathrm{ClN}_{3} \mathrm{O}_{3}$

$M_{r}=321.76$

Triclinic, $P \overline{1}$

Hall symbol: -P 1

$a=8.3007(5) \AA$

$b=12.2295(6) \AA$

$c=16.5605(10) \AA$

$\alpha=103.420(4)^{\circ}$

$\beta=95.561(3)^{\circ}$

$\gamma=106.758(3)^{\circ}$

$V=1541.35(15) \AA^{3}$

\section{Data collection}

Bruker-Nonius KappaCCD area-detector diffractometer

Radiation source: Bruker Nonius FR591 rotating anode

$10 \mathrm{~cm}$ confocal mirrors monochromator

Detector resolution: 9.091 pixels $\mathrm{mm}^{-1}$

$\varphi$ and $\omega$ scans

Absorption correction: multi-scan

(SADABS; Sheldrick, 1997b)

Refinement

Refinement on $F^{2}$

Least-squares matrix: full

$R\left[F^{2}>2 \sigma\left(F^{2}\right)\right]=0.057$

$w R\left(F^{2}\right)=0.139$

$S=1.04$

6038 reflections

418 parameters

5 restraints

Primary atom site location: structure-invariant direct methods
$Z=4$

$F(000)=672$

$D_{\mathrm{x}}=1.387 \mathrm{Mg} \mathrm{m}^{-3}$

Melting point: $375 \mathrm{~K}$

Mo $K \alpha$ radiation, $\lambda=0.71073 \AA$

Cell parameters from 6811 reflections

$\theta=2.9-27.5^{\circ}$

$\mu=0.26 \mathrm{~mm}^{-1}$

$T=120 \mathrm{~K}$

Plate, colourless

$0.36 \times 0.30 \times 0.04 \mathrm{~mm}$

$T_{\min }=0.911, T_{\max }=0.990$

30632 measured reflections

6038 independent reflections

3622 reflections with $I>2 \sigma(I)$

$R_{\text {int }}=0.098$

$\theta_{\max }=26.0^{\circ}, \theta_{\min }=2.9^{\circ}$

$h=-10 \rightarrow 10$

$k=-15 \rightarrow 15$

$l=-20 \rightarrow 20$

Secondary atom site location: difference Fourier map

Hydrogen site location: inferred from neighbouring sites

H-atom parameters constrained

$w=1 /\left[\sigma^{2}\left(F_{\mathrm{o}}^{2}\right)+(0.0645 P)^{2}+0.076 P\right]$

where $P=\left(F_{\mathrm{o}}{ }^{2}+2 F_{\mathrm{c}}{ }^{2}\right) / 3$

$(\Delta / \sigma)_{\max }<0.001$

$\Delta \rho_{\max }=0.29{\mathrm{e} \AA^{-3}}^{-0.34}$

$\Delta \rho_{\min }=-0.34$ e $\AA^{-3}$ 
Special details

Geometry. Least-squares planes $(x, y, z$ in crystal coordinates $)$ and deviations from them $(*$ indicates atom used to define plane)

$-6.8962(0.0067) x+4.9846(0.0145) y-7.8315(0.0209) z=1.6003(0.0120)$

* $-0.0004(0.0016) \mathrm{N} 1 \mathrm{~A} * 0.0000(0.0016) \mathrm{C} 2 \mathrm{~A} * 0.0003(0.0016) \mathrm{N} 3 \mathrm{~A} *{ }_{-0} 0.0006(0.0016) \mathrm{C} 4 \mathrm{~A} * 0.0006(0.0017) \mathrm{C} 5 \mathrm{~A}$

Rms deviation of fitted atoms $=0.0004$

$-2.8599(0.0085) x-3.1842(0.0131) y+15.8848(0.0052) z=0.0246(0.0064)$

Angle to previous plane (with approximate e.s.d.) $=76.46(0.09)$

* $-0.0124(0.0019) \mathrm{C} 32 \mathrm{~A} * 0.0090$ (0.0019) C33A * 0.0041 (0.0019) C34A * $-0.0137(0.0019) \mathrm{C} 35 \mathrm{~A} * 0.0100(0.0019)$ $\mathrm{C} 36 \mathrm{~A} * 0.0031(0.0019) \mathrm{C} 37 \mathrm{~A}$

Rms deviation of fitted atoms $=0.0096$

$7.1630(0.0060) x-4.4739(0.0148) y+6.9396(0.0214) z=6.8216(0.0014)$

Angle to previous plane (with approximate e.s.d.) $=80.19(0.09)$

$* 0.0002(0.0017) \mathrm{N} 1 \mathrm{~B} *-0.0002(0.0017) \mathrm{C} 2 \mathrm{~B} * 0.0002(0.0016) \mathrm{N} 3 \mathrm{~B} * 0.0000(0.0016) \mathrm{C} 4 \mathrm{~B} *-0.0001(0.0017) \mathrm{C} 5 \mathrm{~B}$

Rms deviation of fitted atoms $=0.0002$

$-2.3192(0.0090) x-3.5959(0.0138) y+16.1123(0.0044) z=1.2353(0.0111)$

Angle to previous plane (with approximate e.s.d.) $=76.31(0.10)$

* $0.0029(0.0019) \mathrm{C} 32 \mathrm{~B} *-0.0043(0.0020) \mathrm{C} 33 \mathrm{~B} * 0.0014(0.0020) \mathrm{C} 34 \mathrm{~B} * 0.0030(0.0020) \mathrm{C} 35 \mathrm{~B} *-0.0043(0.0020)$ $\mathrm{C} 36 \mathrm{~B} * 0.0014(0.0020) \mathrm{C} 37 \mathrm{~B}$

Rms deviation of fitted atoms $=0.0031$

Fractional atomic coordinates and isotropic or equivalent isotropic displacement parameters $\left(\AA^{2}\right)$

\begin{tabular}{|c|c|c|c|c|c|}
\hline & $x$ & $y$ & $z$ & $U_{\text {iso }} * / U_{\text {eq }}$ & Occ. $(<1)$ \\
\hline N1A & $0.1941(3)$ & $0.9197(2)$ & $0.21017(15)$ & $0.0320(6)$ & \\
\hline $\mathrm{C} 2 \mathrm{~A}$ & 0.1120 & $0.8084(2)$ & $0.21159(18)$ & $0.0291(7)$ & \\
\hline C21A & 0.0110 & $0.7792(3)$ & 0.27785 (19) & $0.0341(7)$ & \\
\hline $\mathrm{H} 21 \mathrm{~A}$ & -0.1016 & 0.7203 & 0.2497 & $0.043^{*}$ & \\
\hline $\mathrm{H} 22 \mathrm{~A}$ & -0.0099 & 0.8520 & 0.3089 & $0.043^{*}$ & \\
\hline $\mathrm{C} 22 \mathrm{~A}$ & $0.0936(4)$ & $0.7298(3)$ & $0.34200(18)$ & $0.0336(7)$ & \\
\hline $\mathrm{H} 23 \mathrm{~A}$ & 0.1042 & 0.6525 & 0.3127 & $0.042 *$ & \\
\hline $\mathrm{H} 24 \mathrm{~A}$ & 0.2098 & 0.7850 & 0.3678 & $0.042 *$ & \\
\hline $\mathrm{C} 23 \mathrm{~A}$ & $-0.0126(4)$ & 0.7134 & 0.4106 & $0.0452(9)$ & \\
\hline $\mathrm{H} 25 \mathrm{~A}$ & -0.0219 & 0.7912 & 0.4397 & $0.056^{*}$ & \\
\hline $\mathrm{H} 26 \mathrm{~A}$ & -0.1294 & 0.6598 & 0.3840 & $0.056^{*}$ & \\
\hline $\mathrm{C} 24 \mathrm{~A}$ & $0.0598(5)$ & $0.6628(3)$ & $0.4755(2)$ & $0.0473(9)$ & \\
\hline $\mathrm{H} 27 \mathrm{~A}$ & 0.0520 & 0.5806 & 0.4490 & $0.059 *$ & \\
\hline $\mathrm{H} 28 \mathrm{~A}$ & -0.0055 & 0.6649 & 0.5217 & $0.059 *$ & \\
\hline $\mathrm{H} 29 \mathrm{~A}$ & 0.1798 & 0.7103 & 0.4977 & $0.059 *$ & \\
\hline N3A & $0.1292(3)$ & 0.72747 (19) & $0.14485(14)$ & $0.0260(5)$ & \\
\hline C31A & $0.0640(4)$ & $0.5979(2)$ & $0.12824(19)$ & $0.0281(7)$ & \\
\hline $\mathrm{H} 31 \mathrm{~A}$ & 0.0088 & 0.5623 & 0.0683 & $0.035^{*}$ & \\
\hline $\mathrm{H} 32 \mathrm{~A}$ & -0.0239 & 0.5769 & 0.1634 & $0.035^{*}$ & \\
\hline C32A & $0.2046(3)$ & $0.5464(2)$ & $0.14713(17)$ & $0.0244(6)$ & \\
\hline $\mathrm{C} 33 \mathrm{~A}$ & $0.1713(4)$ & $0.4240(2)$ & $0.11796(17)$ & $0.0280(7)$ & \\
\hline $\mathrm{H} 33 \mathrm{~A}$ & 0.0601 & 0.3748 & 0.0892 & $0.035^{*}$ & \\
\hline C34A & 0.2970 & $0.3735(2)$ & $0.13014(17)$ & $0.0278(7)$ & \\
\hline H34A & 0.2740 & 0.2903 & 0.1099 & $0.035^{*}$ & \\
\hline $\mathrm{C} 35 \mathrm{~A}$ & 0.4576 & $0.4473(2)$ & $0.17274(17)$ & $0.0256(6)$ & \\
\hline $\mathrm{C} 36 \mathrm{~A}$ & $0.4929(4)$ & $0.5679(2)$ & $0.20476(18)$ & $0.0286(7)$ & \\
\hline
\end{tabular}




\begin{tabular}{|c|c|c|c|c|c|}
\hline H36A & 0.6029 & 0.6164 & 0.2356 & $0.036^{*}$ & \\
\hline C37A & $0.3659(3)$ & $0.6167(2)$ & $0.19124(17)$ & $0.0273(7)$ & \\
\hline H37A & 0.3892 & 0.6999 & 0.2125 & $0.034^{*}$ & \\
\hline N31A & $0.5943(3)$ & $0.3952(2)$ & $0.18395(15)$ & $0.0306(6)$ & \\
\hline $\mathrm{O} 31 \mathrm{~A}$ & $0.5619(3)$ & $0.28834(18)$ & $0.15276(14)$ & $0.0411(6)$ & \\
\hline $\mathrm{O} 32 \mathrm{~A}$ & 0.7355 & $0.46121(18)$ & $0.22401(13)$ & $0.0389(5)$ & \\
\hline C4A & $0.2293(3)$ & $0.7895(2)$ & $0.09634(18)$ & $0.0270(7)$ & \\
\hline C41A & $0.2801(4)$ & $0.7385(3)$ & 0.01985 (19) & $0.0311(7)$ & \\
\hline $\mathrm{H} 41 \mathrm{~A}$ & 0.3513 & 0.7921 & -0.0052 & $0.039 *$ & \\
\hline $\mathrm{O} 41 \mathrm{~A}$ & 0.2410 & $0.63322(18)$ & $-0.01605(13)$ & $0.0373(5)$ & \\
\hline C5A & $0.2643(4)$ & $0.9064(2)$ & 0.13975 (19) & $0.0307(7)$ & \\
\hline $\mathrm{Cl5A}$ & $0.38198(10)$ & $1.02856(6)$ & $0.11284(5)$ & $0.0410(2)$ & \\
\hline N1B & $0.6628(3)$ & $-0.1150(2)$ & $0.22474(15)$ & $0.0313(6)$ & \\
\hline $\mathrm{C} 2 \mathrm{~B}$ & $0.6981(4)$ & $0.0002(2)$ & $0.26254(18)$ & $0.0300(7)$ & \\
\hline $\mathrm{C} 21 \mathrm{~B}$ & $0.6407(5)$ & $0.0477(3)$ & $0.3422(2)$ & $0.0454(9)$ & \\
\hline $\mathrm{H} 21 \mathrm{~B}$ & 0.5462 & 0.0782 & 0.3284 & $0.057^{*}$ & 0.50 \\
\hline $\mathrm{H} 22 \mathrm{~B}$ & 0.7364 & 0.1153 & 0.3790 & $0.057^{*}$ & 0.50 \\
\hline $\mathrm{H} 21 \mathrm{C}$ & 0.5147 & 0.0136 & 0.3336 & $0.057^{*}$ & 0.50 \\
\hline $\mathrm{H} 22 \mathrm{C}$ & 0.6689 & 0.1346 & 0.3522 & $0.057^{*}$ & 0.50 \\
\hline $\mathrm{C} 22 \mathrm{~B}$ & $0.5810(7)$ & $-0.0426(4)$ & $0.3902(3)$ & $0.0548(14)$ & 0.75 \\
\hline $\mathrm{H} 23 \mathrm{~B}$ & 0.5204 & -0.0093 & 0.4332 & $0.068^{*}$ & 0.75 \\
\hline $\mathrm{H} 24 \mathrm{~B}$ & 0.4953 & -0.1133 & 0.3502 & $0.068^{*}$ & 0.75 \\
\hline $\mathrm{C} 22 \mathrm{C}$ & $0.7160(19)$ & $0.0238(10)$ & $0.4224(7)$ & $0.050(4)$ & 0.25 \\
\hline $\mathrm{H} 23 \mathrm{C}$ & 0.8403 & 0.0667 & 0.4331 & $0.062 *$ & 0.25 \\
\hline $\mathrm{H} 24 \mathrm{C}$ & 0.6695 & 0.0639 & 0.4692 & $0.062 *$ & 0.25 \\
\hline $\mathrm{C} 23 \mathrm{~B}$ & $0.6998(7)$ & $-0.0824(5)$ & 0.4313 & 0.0957 (18) & \\
\hline $\mathrm{H} 25 \mathrm{~B}$ & 0.7781 & -0.0152 & 0.4771 & $0.120 *$ & 0.50 \\
\hline H26B & 0.7690 & -0.1103 & 0.3907 & $0.120 *$ & 0.50 \\
\hline $\mathrm{H} 25 \mathrm{C}$ & 0.7749 & -0.1123 & 0.3953 & $0.120 *$ & 0.50 \\
\hline $\mathrm{H} 26 \mathrm{C}$ & 0.5814 & -0.1311 & 0.4036 & $0.120 *$ & 0.50 \\
\hline $\mathrm{C} 24 \mathrm{~B}$ & $0.6138(14)$ & $-0.1835(8)$ & $0.4688(7)$ & $0.076(3)$ & 0.50 \\
\hline $\mathrm{H} 27 \mathrm{~B}$ & 0.5378 & -0.1587 & 0.5055 & $0.096^{*}$ & 0.50 \\
\hline H28B & 0.7016 & -0.2026 & 0.5016 & $0.096^{*}$ & 0.50 \\
\hline H29B & 0.5473 & -0.2538 & 0.4231 & $0.096^{*}$ & 0.50 \\
\hline $\mathrm{C} 24 \mathrm{C}$ & $0.7279(15)$ & $-0.1186(9)$ & $0.5099(5)$ & $0.077(3)$ & 0.50 \\
\hline $\mathrm{H} 27 \mathrm{C}$ & 0.8509 & -0.0945 & 0.5311 & $0.096^{*}$ & 0.50 \\
\hline $\mathrm{H} 28 \mathrm{C}$ & 0.6787 & -0.2050 & 0.4979 & $0.096^{*}$ & 0.50 \\
\hline $\mathrm{H} 29 \mathrm{C}$ & 0.6728 & -0.0802 & 0.5525 & $0.096^{*}$ & 0.50 \\
\hline N3B & 0.7853 & 0.07047 (19) & $0.21787(14)$ & $0.0272(6)$ & \\
\hline $\mathrm{C} 31 \mathrm{~B}$ & $0.8436(4)$ & $0.2000(2)$ & $0.24006(19)$ & $0.0315(7)$ & \\
\hline H31B & 0.7715 & 0.2295 & 0.2783 & $0.039 *$ & \\
\hline H32B & 0.8270 & 0.2255 & 0.1882 & $0.039 *$ & \\
\hline $\mathrm{C} 32 \mathrm{~B}$ & $1.0288(4)$ & $0.2568(2)$ & $0.28223(17)$ & $0.0255(6)$ & \\
\hline C33B & $1.0880(4)$ & $0.3795(2)$ & $0.31769(18)$ & $0.0315(7)$ & \\
\hline H33B & 1.0113 & 0.4238 & 0.3160 & $0.039 *$ & \\
\hline C34B & $1.2568(4)$ & $0.4371(3)$ & $0.35520(18)$ & $0.0325(7)$ & \\
\hline H34B & 1.2970 & 0.5205 & 0.3798 & $0.041 *$ & \\
\hline C35B & $1.3661(4)$ & $0.3708(3)$ & $0.35625(17)$ & $0.0310(7)$ & \\
\hline
\end{tabular}




$\begin{array}{lllll}\text { C36B } & 1.3121(4) & 0.2497(3) & 0.32099(19) & 0.0346(7) \\ \text { H36B } & 1.3899 & 0.2060 & 0.3219 & 0.043^{*} \\ \text { C37B } & 1.1418(4) & 0.1932(3) & 0.28422(19) & 0.0329(7) \\ \text { H37B } & 1.1022 & 0.1097 & 0.2601 & 0.041^{*} \\ \text { N31B } & 1.5466(3) & 0.4314(3) & 0.39535(16) & 0.0411(7) \\ \text { O31B } & 1.5914(3) & 0.5382(2) & 0.42867(15) & 0.0552(7) \\ \text { O32B } & 1.6441(3) & 0.3719(2) & 0.39210(16) & 0.0591(7) \\ \text { C4B } & 0.8079(3) & -0.0049(2) & 0.14593(17) & 0.0266(7) \\ \text { C41B } & 0.8932(4) & 0.0287(3) & 0.07954(19) & 0.0342(7) \\ \text { H41B } & 0.8993 & -0.0338 & 0.0349 & 0.043^{*} \\ \text { O41B } & 0.9582(3) & 0.12965(18) & 0.07510(13) & 0.0424(6) \\ \text { C5B } & 0.7303(3) & -0.1171(2) & 0.15369(17) & 0.0274(7) \\ \text { C15B } & 0.71564(10) & -0.24842(6) & 0.08330(5) & 0.0357(2) \\ \end{array}$

Atomic displacement parameters $\left(\AA^{2}\right)$

\begin{tabular}{|c|c|c|c|c|c|c|}
\hline & $U^{11}$ & $U^{22}$ & $U^{33}$ & $U^{12}$ & $U^{13}$ & $U^{23}$ \\
\hline N1A & $0.0331(15)$ & $0.0262(14)$ & $0.0401(16)$ & $0.0145(11)$ & $0.0045(12)$ & $0.0103(11)$ \\
\hline $\mathrm{C} 2 \mathrm{~A}$ & $0.0258(17)$ & $0.0268(16)$ & $0.0372(18)$ & $0.0137(13)$ & $0.0027(13)$ & $0.0081(14)$ \\
\hline $\mathrm{C} 21 \mathrm{~A}$ & $0.0330(18)$ & $0.0349(17)$ & $0.0386(18)$ & 0.0177 (14) & $0.0104(15)$ & $0.0079(14)$ \\
\hline $\mathrm{C} 22 \mathrm{~A}$ & $0.0310(18)$ & $0.0322(17)$ & $0.0368(18)$ & $0.0106(14)$ & $0.0047(14)$ & $0.0076(14)$ \\
\hline $\mathrm{C} 23 \mathrm{~A}$ & $0.046(2)$ & $0.051(2)$ & $0.046(2)$ & $0.0214(17)$ & $0.0163(17)$ & $0.0166(17)$ \\
\hline $\mathrm{C} 24 \mathrm{~A}$ & $0.064(2)$ & $0.043(2)$ & $0.037(2)$ & $0.0204(18)$ & $0.0147(17)$ & $0.0091(16)$ \\
\hline N3A & $0.0208(13)$ & $0.0216(12)$ & $0.0368(14)$ & $0.0084(10)$ & $0.0040(11)$ & $0.0089(11)$ \\
\hline C31A & $0.0244(16)$ & $0.0208(15)$ & $0.0366(17)$ & $0.0043(12)$ & $0.0047(13)$ & 0.0067 (13) \\
\hline C32A & $0.0247(16)$ & $0.0205(14)$ & $0.0307(16)$ & $0.0071(12)$ & $0.0088(13)$ & $0.0107(12)$ \\
\hline C33A & $0.0266(17)$ & $0.0240(15)$ & $0.0295(16)$ & $0.0044(13)$ & $0.0007(13)$ & $0.0060(13)$ \\
\hline C34A & $0.0302(17)$ & $0.0236(15)$ & $0.0308(17)$ & $0.0094(13)$ & $0.0060(13)$ & $0.0084(13)$ \\
\hline C35A & $0.0228(16)$ & $0.0262(15)$ & $0.0329(16)$ & $0.0106(13)$ & $0.0085(13)$ & $0.0128(13)$ \\
\hline $\mathrm{C} 36 \mathrm{~A}$ & $0.0238(16)$ & $0.0258(15)$ & $0.0342(17)$ & $0.0048(13)$ & $0.0022(13)$ & $0.0096(13)$ \\
\hline C37A & $0.0264(17)$ & $0.0185(14)$ & $0.0331(17)$ & $0.0052(12)$ & $0.0024(13)$ & $0.0036(12)$ \\
\hline N31A & $0.0307(15)$ & $0.0309(15)$ & $0.0372(15)$ & $0.0138(12)$ & $0.0091(12)$ & $0.0161(12)$ \\
\hline O31A & $0.0392(13)$ & $0.0263(12)$ & $0.0652(16)$ & $0.0170(10)$ & $0.0119(11)$ & $0.0170(11)$ \\
\hline O32A & $0.0270(13)$ & $0.0405(13)$ & $0.0488(14)$ & $0.0119(10)$ & $-0.0011(10)$ & $0.0133(11)$ \\
\hline $\mathrm{C} 4 \mathrm{~A}$ & $0.0207(15)$ & $0.0256(15)$ & $0.0360(18)$ & $0.0069(12)$ & $0.0036(13)$ & $0.0124(13)$ \\
\hline C41A & $0.0225(16)$ & $0.0327(18)$ & $0.0392(19)$ & $0.0073(13)$ & $0.0012(14)$ & $0.0156(15)$ \\
\hline O41A & $0.0344(13)$ & $0.0310(12)$ & $0.0399(13)$ & $0.0063(10)$ & $0.0046(10)$ & $0.0032(10)$ \\
\hline $\mathrm{C} 5 \mathrm{~A}$ & $0.0289(17)$ & $0.0207(15)$ & $0.0433(19)$ & $0.0079(13)$ & $-0.0002(14)$ & $0.0130(14)$ \\
\hline $\mathrm{C} 15 \mathrm{~A}$ & $0.0365(5)$ & $0.0269(4)$ & $0.0585(5)$ & $0.0048(3)$ & $0.0017(4)$ & $0.0192(4)$ \\
\hline N1B & $0.0314(15)$ & $0.0275(14)$ & $0.0336(15)$ & $0.0085(11)$ & $0.0059(12)$ & $0.0069(11)$ \\
\hline $\mathrm{C} 2 \mathrm{~B}$ & $0.0283(17)$ & $0.0290(17)$ & $0.0329(17)$ & $0.0103(13)$ & $0.0039(14)$ & $0.0083(14)$ \\
\hline $\mathrm{C} 21 \mathrm{~B}$ & $0.063(2)$ & $0.0383(19)$ & $0.043(2)$ & $0.0260(18)$ & $0.0199(18)$ & $0.0098(16)$ \\
\hline $\mathrm{C} 22 \mathrm{~B}$ & $0.079(4)$ & $0.040(3)$ & $0.060(3)$ & $0.028(3)$ & $0.041(3)$ & $0.019(3)$ \\
\hline $\mathrm{C} 22 \mathrm{C}$ & $0.065(11)$ & $0.023(7)$ & $0.046(9)$ & $-0.002(7)$ & $0.019(8)$ & $-0.003(6)$ \\
\hline $\mathrm{C} 23 \mathrm{~B}$ & $0.156(5)$ & $0.123(4)$ & $0.071(3)$ & $0.104(4)$ & $0.058(3)$ & 0.055 \\
\hline $\mathrm{C} 24 \mathrm{~B}$ & $0.117(10)$ & $0.084(8)$ & $0.079(8)$ & $0.073(7)$ & $0.059(7)$ & $0.052(6)$ \\
\hline $\mathrm{C} 24 \mathrm{C}$ & $0.125(10)$ & $0.092(8)$ & $0.049(6)$ & $0.068(7)$ & $0.036(6)$ & $0.033(6)$ \\
\hline N3B & $0.0242(13)$ & $0.0220(12)$ & $0.0336(14)$ & $0.0086(10)$ & -0.0008 & $0.0048(11)$ \\
\hline
\end{tabular}




$\begin{array}{lllllll}\text { C31B } & 0.0316(18) & 0.0231(15) & 0.0405(18) & 0.0128(13) & 0.0006(14) & 0.0070(13) \\ \text { C32B } & 0.0293(17) & 0.0260(15) & 0.0226(15) & 0.0100(13) & 0.0032(12) & 0.0084(12) \\ \text { C33B } & 0.0328(18) & 0.0269(16) & 0.0384(18) & 0.0136(14) & 0.0083(14) & 0.0101(14) \\ \text { C34B } & 0.0360(19) & 0.0247(15) & 0.0344(18) & 0.0088(14) & 0.0073(14) & 0.0042(13) \\ \text { C35B } & 0.0261(17) & 0.0408(18) & 0.0256(16) & 0.0089(14) & 0.0037(13) & 0.0106(14) \\ \text { C36B } & 0.0330(19) & 0.0356(18) & 0.0393(19) & 0.0169(15) & 0.0024(15) & 0.0122(14) \\ \text { C37B } & 0.0351(19) & 0.0239(15) & 0.0381(18) & 0.0115(14) & -0.0008(14) & 0.0057(13) \\ \text { N31B } & 0.0331(17) & 0.0472(18) & 0.0363(16) & 0.0033(15) & 0.0035(13) & 0.0119(14) \\ \text { O31B } & 0.0392(14) & 0.0497(16) & 0.0583(16) & -0.0034(12) & 0.0022(12) & 0.0049(13) \\ \text { O32B } & 0.0347(15) & 0.0721(18) & 0.0692(18) & 0.0201(14) & -0.0042(12) & 0.0184(14) \\ \text { C4B } & 0.0228(16) & 0.0262(16) & 0.0295(17) & 0.0079(12) & 0.0018(13) & 0.0065(13) \\ \text { C41B } & 0.0315(18) & 0.0335(18) & 0.0375(19) & 0.0104(14) & 0.0036(14) & 0.0103(14) \\ \text { O41B } & 0.0462(14) & 0.0311(12) & 0.0479(14) & 0.0051(10) & 0.0115(11) & 0.0148(10) \\ \text { C5B } & 0.0257(16) & 0.0254(15) & 0.0262(16) & 0.0074(13) & -0.0013(13) & 0.0010(12) \\ \text { C15B } & 0.0403(5) & 0.0268(4) & 0.0343(4) & 0.0076(3) & 0.0046(3) & 0.0026(3) \\ & & & & & & \end{array}$

Geometric parameters $\left(\AA,{ }^{\circ}\right)$

\begin{tabular}{llll}
\hline $\mathrm{N} 1 \mathrm{~A}-\mathrm{C} 2 \mathrm{~A}$ & $1.341(3)$ & $\mathrm{C} 2 \mathrm{~B}-\mathrm{C} 21 \mathrm{~B}$ & $1.494(4)$ \\
$\mathrm{N} 1 \mathrm{~A}-\mathrm{C} 5 \mathrm{~A}$ & $1.351(4)$ & $\mathrm{C} 21 \mathrm{~B}-\mathrm{C} 22 \mathrm{~B}$ & $1.504(5)$ \\
$\mathrm{C} 2 \mathrm{~A}-\mathrm{N} 3 \mathrm{~A}$ & $1.351(4)$ & $\mathrm{C} 21 \mathrm{~B}-\mathrm{H} 21 \mathrm{~B}$ & 0.99 \\
$\mathrm{C} 2 \mathrm{~A}-\mathrm{C} 21 \mathrm{~A}$ & $1.491(4)$ & $\mathrm{C} 21 \mathrm{~B}-\mathrm{H} 22 \mathrm{~B}$ & 0.99 \\
$\mathrm{C} 21 \mathrm{~A}-\mathrm{C} 22 \mathrm{~A}$ & $1.537(4)$ & $\mathrm{C} 22 \mathrm{~B}-\mathrm{C} 23 \mathrm{~B}$ & $1.405(6)$ \\
$\mathrm{C} 21 \mathrm{~A}-\mathrm{H} 21 \mathrm{~A}$ & 0.99 & $\mathrm{C} 22 \mathrm{~B}-\mathrm{H} 23 \mathrm{~B}$ & 0.99 \\
$\mathrm{C} 21 \mathrm{~A}-\mathrm{H} 22 \mathrm{~A}$ & 0.99 & $\mathrm{C} 22 \mathrm{~B}-\mathrm{H} 24 \mathrm{~B}$ & 0.99 \\
$\mathrm{C} 22 \mathrm{~A}-\mathrm{C} 23 \mathrm{~A}$ & $1.518(4)$ & $\mathrm{C} 23 \mathrm{~B}-\mathrm{C} 24 \mathrm{~B}$ & $1.534(9)$ \\
$\mathrm{C} 22 \mathrm{~A}-\mathrm{H} 23 \mathrm{~A}$ & 0.99 & $\mathrm{C} 23 \mathrm{~B}-\mathrm{H} 25 \mathrm{~B}$ & 0.99 \\
$\mathrm{C} 22 \mathrm{~A}-\mathrm{H} 24 \mathrm{~A}$ & 0.99 & $\mathrm{C} 23 \mathrm{~B}-\mathrm{H} 26 \mathrm{~B}$ & 0.99 \\
$\mathrm{C} 23 \mathrm{~A}-\mathrm{C} 24 \mathrm{~A}$ & $1.515(4)$ & $\mathrm{C} 24 \mathrm{~B}-\mathrm{H} 27 \mathrm{~B}$ & 0.98 \\
$\mathrm{C} 23 \mathrm{~A}-\mathrm{H} 25 \mathrm{~A}$ & 0.99 & $\mathrm{C} 24 \mathrm{~B}-\mathrm{H} 28 \mathrm{~B}$ & 0.98 \\
$\mathrm{C} 23 \mathrm{~A}-\mathrm{H} 26 \mathrm{~A}$ & 0.99 & $\mathrm{C} 24 \mathrm{~B}-\mathrm{H} 29 \mathrm{~B}$ & 0.98 \\
$\mathrm{C} 24 \mathrm{~A}-\mathrm{H} 27 \mathrm{~A}$ & 0.98 & $\mathrm{C} 22 \mathrm{C}-\mathrm{H} 23 \mathrm{C}$ & 0.99 \\
$\mathrm{C} 24 \mathrm{~A}-\mathrm{H} 28 \mathrm{~A}$ & 0.98 & $\mathrm{C} 22 \mathrm{C}-\mathrm{H} 24 \mathrm{C}$ & 0.99 \\
$\mathrm{C} 24 \mathrm{~A}-\mathrm{H} 29 \mathrm{~A}$ & 0.98 & $\mathrm{C} 24 \mathrm{C}-\mathrm{H} 27 \mathrm{C}$ & 0.98 \\
$\mathrm{~N} 3 \mathrm{~A}-\mathrm{C} 4 \mathrm{~A}$ & $1.400(3)$ & $\mathrm{C} 24 \mathrm{C}-\mathrm{H} 28 \mathrm{C}$ & 0.98 \\
$\mathrm{~N} 3 \mathrm{~A}-\mathrm{C} 31 \mathrm{~A}$ & $1.467(3)$ & $\mathrm{C} 24 \mathrm{C}-\mathrm{H} 29 \mathrm{C}$ & 0.98 \\
$\mathrm{C} 31 \mathrm{~A}-\mathrm{C} 32 \mathrm{~A}$ & $1.518(4)$ & $\mathrm{N} 3 \mathrm{~B}-\mathrm{C} 4 \mathrm{~B}$ & $1.392(3)$ \\
$\mathrm{C} 31 \mathrm{~A}-\mathrm{H} 31 \mathrm{~A}$ & 0.99 & $\mathrm{~N} 3 \mathrm{~B}-\mathrm{C} 31 \mathrm{~B}$ & $1.460(3)$ \\
$\mathrm{C} 31 \mathrm{~A}-\mathrm{H} 32 \mathrm{~A}$ & 0.99 & $\mathrm{C} 31 \mathrm{~B}-\mathrm{C} 32 \mathrm{~B}$ & $1.511(4)$ \\
$\mathrm{C} 32 \mathrm{~A}-\mathrm{C} 37 \mathrm{~A}$ & $1.386(4)$ & $\mathrm{C} 31 \mathrm{~B}-\mathrm{H} 31 \mathrm{~B}$ & 0.99 \\
$\mathrm{C} 32 \mathrm{~A}-\mathrm{C} 33 \mathrm{~A}$ & $1.395(4)$ & $\mathrm{C} 31 \mathrm{~B}-\mathrm{H} 32 \mathrm{~B}$ & 0.99 \\
$\mathrm{C} 33 \mathrm{~A}-\mathrm{C} 34 \mathrm{~A}$ & $1.378(4)$ & $\mathrm{C} 32 \mathrm{~B}-\mathrm{C} 37 \mathrm{~B}$ & $1.383(4)$ \\
$\mathrm{C} 33 \mathrm{~A}-\mathrm{H} 33 \mathrm{~A}$ & 0.95 & $\mathrm{C} 32 \mathrm{~B}-\mathrm{C} 33 \mathrm{~B}$ & $1.396(4)$ \\
$\mathrm{C} 34 \mathrm{~A}-\mathrm{C} 35 \mathrm{~A}$ & $1.385(4)$ & $\mathrm{C} 33 \mathrm{~B}-\mathrm{C} 34 \mathrm{~B}$ & $1.380(4)$ \\
$\mathrm{C} 34 \mathrm{~A}-\mathrm{H} 34 \mathrm{~A}$ & 0.95 & $\mathrm{C} 33 \mathrm{~B}-\mathrm{H} 33 \mathrm{~B}$ & 0.95 \\
$\mathrm{C} 35 \mathrm{~A}-\mathrm{C} 36 \mathrm{~A}$ & $1.378(4)$ & $\mathrm{C} 34 \mathrm{~B}-\mathrm{C} 35 \mathrm{~B}$ & $1.381(4)$ \\
$\mathrm{C} 35 \mathrm{~A}-\mathrm{N} 31 \mathrm{~A}$ & $1.469(3)$ & $\mathrm{C} 35 \mathrm{~B}-\mathrm{B}-\mathrm{C} 36 \mathrm{~B}$ & $1.379(4)$ \\
$\mathrm{C} 36 \mathrm{~A}-\mathrm{C} 37 \mathrm{~A}$ & $1.378(4)$ & &
\end{tabular}




\begin{tabular}{|c|c|c|c|}
\hline $\mathrm{C} 36 \mathrm{~A}-\mathrm{H} 36 \mathrm{~A}$ & 0.95 & $\mathrm{C} 35 \mathrm{~B}-\mathrm{N} 31 \mathrm{~B}$ & $1.470(4)$ \\
\hline $\mathrm{C} 37 \mathrm{~A}-\mathrm{H} 37 \mathrm{~A}$ & 0.95 & $\mathrm{C} 36 \mathrm{~B}-\mathrm{C} 37 \mathrm{~B}$ & $1.386(4)$ \\
\hline $\mathrm{N} 31 \mathrm{~A}-\mathrm{O} 31 \mathrm{~A}$ & $1.227(3)$ & $\mathrm{C} 36 \mathrm{~B}-\mathrm{H} 36 \mathrm{~B}$ & 0.95 \\
\hline $\mathrm{N} 31 \mathrm{~A}-\mathrm{O} 32 \mathrm{~A}$ & $1.233(3)$ & $\mathrm{C} 37 \mathrm{~B}-\mathrm{H} 37 \mathrm{~B}$ & 0.95 \\
\hline $\mathrm{C} 4 \mathrm{~A}-\mathrm{C} 5 \mathrm{~A}$ & $1.373(4)$ & $\mathrm{N} 31 \mathrm{~B}-\mathrm{O} 31 \mathrm{~B}$ & $1.222(3)$ \\
\hline $\mathrm{C} 4 \mathrm{~A}-\mathrm{C} 41 \mathrm{~A}$ & $1.436(4)$ & $\mathrm{N} 31 \mathrm{~B}-\mathrm{O} 32 \mathrm{~B}$ & $1.233(3)$ \\
\hline $\mathrm{C} 41 \mathrm{~A}-\mathrm{O} 41 \mathrm{~A}$ & $1.217(3)$ & $\mathrm{C} 4 \mathrm{~B}-\mathrm{C} 5 \mathrm{~B}$ & $1.379(4)$ \\
\hline $\mathrm{C} 41 \mathrm{~A}-\mathrm{H} 41 \mathrm{~A}$ & 0.95 & $\mathrm{C} 4 \mathrm{~B}-\mathrm{C} 41 \mathrm{~B}$ & $1.439(4)$ \\
\hline $\mathrm{C} 5 \mathrm{~A}-\mathrm{Cl} 5 \mathrm{~A}$ & $1.714(3)$ & $\mathrm{C} 41 \mathrm{~B}-\mathrm{O} 41 \mathrm{~B}$ & $1.219(3)$ \\
\hline $\mathrm{N} 1 \mathrm{~B}-\mathrm{C} 2 \mathrm{~B}$ & $1.334(3)$ & $\mathrm{C} 41 \mathrm{~B}-\mathrm{H} 41 \mathrm{~B}$ & 0.95 \\
\hline $\mathrm{N} 1 \mathrm{~B}-\mathrm{C} 5 \mathrm{~B}$ & $1.349(4)$ & $\mathrm{C} 5 \mathrm{~B}-\mathrm{Cl} 5 \mathrm{~B}$ & $1.716(3)$ \\
\hline $\mathrm{C} 2 \mathrm{~B}-\mathrm{N} 3 \mathrm{~B}$ & $1.360(3)$ & & \\
\hline $\mathrm{C} 2 \mathrm{~A}-\mathrm{N} 1 \mathrm{~A}-\mathrm{C} 5 \mathrm{~A}$ & $104.4(2)$ & $\mathrm{C} 4 \mathrm{~A}-\mathrm{C} 5 \mathrm{~A}-\mathrm{Cl} 5 \mathrm{~A}$ & $126.9(2)$ \\
\hline $\mathrm{N} 1 \mathrm{~A}-\mathrm{C} 2 \mathrm{~A}-\mathrm{N} 3 \mathrm{~A}$ & $111.6(3)$ & $\mathrm{C} 2 \mathrm{~B}-\mathrm{N} 1 \mathrm{~B}-\mathrm{C} 5 \mathrm{~B}$ & $104.4(2)$ \\
\hline $\mathrm{N} 1 \mathrm{~A}-\mathrm{C} 2 \mathrm{~A}-\mathrm{C} 21 \mathrm{~A}$ & $123.6(3)$ & $\mathrm{N} 1 \mathrm{~B}-\mathrm{C} 2 \mathrm{~B}-\mathrm{N} 3 \mathrm{~B}$ & $112.2(2)$ \\
\hline $\mathrm{N} 3 \mathrm{~A}-\mathrm{C} 2 \mathrm{~A}-\mathrm{C} 21 \mathrm{~A}$ & $124.7(2)$ & $\mathrm{N} 1 \mathrm{~B}-\mathrm{C} 2 \mathrm{~B}-\mathrm{C} 21 \mathrm{~B}$ & $124.4(3)$ \\
\hline $\mathrm{C} 2 \mathrm{~A}-\mathrm{C} 21 \mathrm{~A}-\mathrm{C} 22 \mathrm{~A}$ & $115.5(2)$ & $\mathrm{N} 3 \mathrm{~B}-\mathrm{C} 2 \mathrm{~B}-\mathrm{C} 21 \mathrm{~B}$ & $123.3(2)$ \\
\hline $\mathrm{C} 2 \mathrm{~A}-\mathrm{C} 21 \mathrm{~A}-\mathrm{H} 21 \mathrm{~A}$ & 108.4 & $\mathrm{C} 2 \mathrm{~B}-\mathrm{N} 3 \mathrm{~B}-\mathrm{C} 4 \mathrm{~B}$ & $106.8(2)$ \\
\hline $\mathrm{C} 22 \mathrm{~A}-\mathrm{C} 21 \mathrm{~A}-\mathrm{H} 21 \mathrm{~A}$ & 108.4 & $\mathrm{C} 2 \mathrm{~B}-\mathrm{N} 3 \mathrm{~B}-\mathrm{C} 31 \mathrm{~B}$ & $127.0(2)$ \\
\hline $\mathrm{C} 2 \mathrm{~A}-\mathrm{C} 21 \mathrm{~A}-\mathrm{H} 22 \mathrm{~A}$ & 108.4 & $\mathrm{C} 4 \mathrm{~B}-\mathrm{N} 3 \mathrm{~B}-\mathrm{C} 31 \mathrm{~B}$ & $126.1(2)$ \\
\hline $\mathrm{C} 22 \mathrm{~A}-\mathrm{C} 21 \mathrm{~A}-\mathrm{H} 22 \mathrm{~A}$ & 108.4 & $\mathrm{~N} 3 \mathrm{~B}-\mathrm{C} 31 \mathrm{~B}-\mathrm{C} 32 \mathrm{~B}$ & $114.0(2)$ \\
\hline $\mathrm{H} 21 \mathrm{~A}-\mathrm{C} 21 \mathrm{~A}-\mathrm{H} 22 \mathrm{~A}$ & 107.5 & $\mathrm{~N} 3 \mathrm{~B}-\mathrm{C} 31 \mathrm{~B}-\mathrm{H} 31 \mathrm{~B}$ & 108.8 \\
\hline $\mathrm{C} 23 \mathrm{~A}-\mathrm{C} 22 \mathrm{~A}-\mathrm{C} 21 \mathrm{~A}$ & $110.9(2)$ & $\mathrm{C} 32 \mathrm{~B}-\mathrm{C} 31 \mathrm{~B}-\mathrm{H} 31 \mathrm{~B}$ & 108.8 \\
\hline $\mathrm{C} 23 \mathrm{~A}-\mathrm{C} 22 \mathrm{~A}-\mathrm{H} 23 \mathrm{~A}$ & 109.5 & $\mathrm{~N} 3 \mathrm{~B}-\mathrm{C} 31 \mathrm{~B}-\mathrm{H} 32 \mathrm{~B}$ & 108.8 \\
\hline $\mathrm{C} 21 \mathrm{~A}-\mathrm{C} 22 \mathrm{~A}-\mathrm{H} 23 \mathrm{~A}$ & 109.5 & $\mathrm{C} 32 \mathrm{~B}-\mathrm{C} 31 \mathrm{~B}-\mathrm{H} 32 \mathrm{~B}$ & 108.8 \\
\hline $\mathrm{C} 23 \mathrm{~A}-\mathrm{C} 22 \mathrm{~A}-\mathrm{H} 24 \mathrm{~A}$ & 109.5 & $\mathrm{H} 31 \mathrm{~B}-\mathrm{C} 31 \mathrm{~B}-\mathrm{H} 32 \mathrm{~B}$ & 107.7 \\
\hline $\mathrm{C} 21 \mathrm{~A}-\mathrm{C} 22 \mathrm{~A}-\mathrm{H} 24 \mathrm{~A}$ & 109.5 & $\mathrm{C} 37 \mathrm{~B}-\mathrm{C} 32 \mathrm{~B}-\mathrm{C} 33 \mathrm{~B}$ & $119.2(3)$ \\
\hline $\mathrm{H} 23 \mathrm{~A}-\mathrm{C} 22 \mathrm{~A}-\mathrm{H} 24 \mathrm{~A}$ & 108.0 & $\mathrm{C} 37 \mathrm{~B}-\mathrm{C} 32 \mathrm{~B}-\mathrm{C} 31 \mathrm{~B}$ & $122.9(2)$ \\
\hline $\mathrm{C} 24 \mathrm{~A}-\mathrm{C} 23 \mathrm{~A}-\mathrm{C} 22 \mathrm{~A}$ & $113.9(3)$ & $\mathrm{C} 33 \mathrm{~B}-\mathrm{C} 32 \mathrm{~B}-\mathrm{C} 31 \mathrm{~B}$ & $117.8(2)$ \\
\hline $\mathrm{C} 24 \mathrm{~A}-\mathrm{C} 23 \mathrm{~A}-\mathrm{H} 25 \mathrm{~A}$ & 108.8 & $\mathrm{C} 34 \mathrm{~B}-\mathrm{C} 33 \mathrm{~B}-\mathrm{C} 32 \mathrm{~B}$ & $120.7(3)$ \\
\hline $\mathrm{C} 22 \mathrm{~A}-\mathrm{C} 23 \mathrm{~A}-\mathrm{H} 25 \mathrm{~A}$ & 108.8 & $\mathrm{C} 34 \mathrm{~B}-\mathrm{C} 33 \mathrm{~B}-\mathrm{H} 33 \mathrm{~B}$ & 119.7 \\
\hline $\mathrm{C} 24 \mathrm{~A}-\mathrm{C} 23 \mathrm{~A}-\mathrm{H} 26 \mathrm{~A}$ & 108.8 & $\mathrm{C} 32 \mathrm{~B}-\mathrm{C} 33 \mathrm{~B}-\mathrm{H} 33 \mathrm{~B}$ & 119.7 \\
\hline $\mathrm{C} 22 \mathrm{~A}-\mathrm{C} 23 \mathrm{~A}-\mathrm{H} 26 \mathrm{~A}$ & 108.8 & $\mathrm{C} 33 \mathrm{~B}-\mathrm{C} 34 \mathrm{~B}-\mathrm{C} 35 \mathrm{~B}$ & $118.6(3)$ \\
\hline $\mathrm{H} 25 \mathrm{~A}-\mathrm{C} 23 \mathrm{~A}-\mathrm{H} 26 \mathrm{~A}$ & 107.7 & $\mathrm{C} 33 \mathrm{~B}-\mathrm{C} 34 \mathrm{~B}-\mathrm{H} 34 \mathrm{~B}$ & 120.7 \\
\hline $\mathrm{C} 23 \mathrm{~A}-\mathrm{C} 24 \mathrm{~A}-\mathrm{H} 27 \mathrm{~A}$ & 109.5 & $\mathrm{C} 35 \mathrm{~B}-\mathrm{C} 34 \mathrm{~B}-\mathrm{H} 34 \mathrm{~B}$ & 120.7 \\
\hline $\mathrm{C} 23 \mathrm{~A}-\mathrm{C} 24 \mathrm{~A}-\mathrm{H} 28 \mathrm{~A}$ & 109.5 & $\mathrm{C} 36 \mathrm{~B}-\mathrm{C} 35 \mathrm{~B}-\mathrm{C} 34 \mathrm{~B}$ & $122.2(3)$ \\
\hline $\mathrm{H} 27 \mathrm{~A}-\mathrm{C} 24 \mathrm{~A}-\mathrm{H} 28 \mathrm{~A}$ & 109.5 & $\mathrm{C} 36 \mathrm{~B}-\mathrm{C} 35 \mathrm{~B}-\mathrm{N} 31 \mathrm{~B}$ & $118.8(3)$ \\
\hline $\mathrm{C} 23 \mathrm{~A}-\mathrm{C} 24 \mathrm{~A}-\mathrm{H} 29 \mathrm{~A}$ & 109.5 & $\mathrm{C} 34 \mathrm{~B}-\mathrm{C} 35 \mathrm{~B}-\mathrm{N} 31 \mathrm{~B}$ & $119.0(3)$ \\
\hline $\mathrm{H} 27 \mathrm{~A}-\mathrm{C} 24 \mathrm{~A}-\mathrm{H} 29 \mathrm{~A}$ & 109.5 & $\mathrm{C} 35 \mathrm{~B}-\mathrm{C} 36 \mathrm{~B}-\mathrm{C} 37 \mathrm{~B}$ & $118.4(3)$ \\
\hline $\mathrm{H} 28 \mathrm{~A}-\mathrm{C} 24 \mathrm{~A}-\mathrm{H} 29 \mathrm{~A}$ & 109.5 & $\mathrm{C} 35 \mathrm{~B}-\mathrm{C} 36 \mathrm{~B}-\mathrm{H} 36 \mathrm{~B}$ & 120.8 \\
\hline $\mathrm{C} 2 \mathrm{~A}-\mathrm{N} 3 \mathrm{~A}-\mathrm{C} 4 \mathrm{~A}$ & $107.6(2)$ & $\mathrm{C} 37 \mathrm{~B}-\mathrm{C} 36 \mathrm{~B}-\mathrm{H} 36 \mathrm{~B}$ & 120.8 \\
\hline $\mathrm{C} 2 \mathrm{~A}-\mathrm{N} 3 \mathrm{~A}-\mathrm{C} 31 \mathrm{~A}$ & $126.6(2)$ & $\mathrm{C} 32 \mathrm{~B}-\mathrm{C} 37 \mathrm{~B}-\mathrm{C} 36 \mathrm{~B}$ & $120.9(3)$ \\
\hline $\mathrm{C} 4 \mathrm{~A}-\mathrm{N} 3 \mathrm{~A}-\mathrm{C} 31 \mathrm{~A}$ & $125.7(2)$ & $\mathrm{C} 32 \mathrm{~B}-\mathrm{C} 37 \mathrm{~B}-\mathrm{H} 37 \mathrm{~B}$ & 119.6 \\
\hline $\mathrm{N} 3 \mathrm{~A}-\mathrm{C} 31 \mathrm{~A}-\mathrm{C} 32 \mathrm{~A}$ & $112.1(2)$ & $\mathrm{C} 36 \mathrm{~B}-\mathrm{C} 37 \mathrm{~B}-\mathrm{H} 37 \mathrm{~B}$ & 119.6 \\
\hline $\mathrm{N} 3 \mathrm{~A}-\mathrm{C} 31 \mathrm{~A}-\mathrm{H} 31 \mathrm{~A}$ & 109.2 & $\mathrm{O} 31 \mathrm{~B}-\mathrm{N} 31 \mathrm{~B}-\mathrm{O} 32 \mathrm{~B}$ & $123.8(3)$ \\
\hline $\mathrm{C} 32 \mathrm{~A}-\mathrm{C} 31 \mathrm{~A}-\mathrm{H} 31 \mathrm{~A}$ & 109.2 & $\mathrm{O} 31 \mathrm{~B}-\mathrm{N} 31 \mathrm{~B}-\mathrm{C} 35 \mathrm{~B}$ & $118.0(3)$ \\
\hline $\mathrm{N} 3 \mathrm{~A}-\mathrm{C} 31 \mathrm{~A}-\mathrm{H} 32 \mathrm{~A}$ & 109.2 & $\mathrm{O} 32 \mathrm{~B}-\mathrm{N} 31 \mathrm{~B}-\mathrm{C} 35 \mathrm{~B}$ & $118.2(3)$ \\
\hline
\end{tabular}




\begin{tabular}{|c|c|}
\hline $\mathrm{C} 32 \mathrm{~A}-\mathrm{C} 31 \mathrm{~A}-\mathrm{H} 32 \mathrm{~A}$ & 109.2 \\
\hline $\mathrm{H} 31 \mathrm{~A}-\mathrm{C} 31 \mathrm{~A}-\mathrm{H} 32 \mathrm{~A}$ & 107.9 \\
\hline $\mathrm{C} 37 \mathrm{~A}-\mathrm{C} 32 \mathrm{~A}-\mathrm{C} 33 \mathrm{~A}$ & $118.8(3)$ \\
\hline $\mathrm{C} 37 \mathrm{~A}-\mathrm{C} 32 \mathrm{~A}-\mathrm{C} 31 \mathrm{~A}$ & $122.3(2)$ \\
\hline $\mathrm{C} 33 \mathrm{~A}-\mathrm{C} 32 \mathrm{~A}-\mathrm{C} 31 \mathrm{~A}$ & $118.9(2)$ \\
\hline $\mathrm{C} 34 \mathrm{~A}-\mathrm{C} 33 \mathrm{~A}-\mathrm{C} 32 \mathrm{~A}$ & $121.1(3)$ \\
\hline $\mathrm{C} 34 \mathrm{~A}-\mathrm{C} 33 \mathrm{~A}-\mathrm{H} 33 \mathrm{~A}$ & 119.4 \\
\hline $\mathrm{C} 32 \mathrm{~A}-\mathrm{C} 33 \mathrm{~A}-\mathrm{H} 33 \mathrm{~A}$ & 119.4 \\
\hline $\mathrm{C} 33 \mathrm{~A}-\mathrm{C} 34 \mathrm{~A}-\mathrm{C} 35 \mathrm{~A}$ & $118.2(3)$ \\
\hline $\mathrm{C} 33 \mathrm{~A}-\mathrm{C} 34 \mathrm{~A}-\mathrm{H} 34 \mathrm{~A}$ & 120.9 \\
\hline $\mathrm{C} 35 \mathrm{~A}-\mathrm{C} 34 \mathrm{~A}-\mathrm{H} 34 \mathrm{~A}$ & 120.9 \\
\hline $\mathrm{C} 36 \mathrm{~A}-\mathrm{C} 35 \mathrm{~A}-\mathrm{C} 34 \mathrm{~A}$ & $122.0(3)$ \\
\hline $\mathrm{C} 36 \mathrm{~A}-\mathrm{C} 35 \mathrm{~A}-\mathrm{N} 31 \mathrm{~A}$ & $119.1(2)$ \\
\hline $\mathrm{C} 34 \mathrm{~A}-\mathrm{C} 35 \mathrm{~A}-\mathrm{N} 31 \mathrm{~A}$ & $118.9(2)$ \\
\hline $\mathrm{C} 37 \mathrm{~A}-\mathrm{C} 36 \mathrm{~A}-\mathrm{C} 35 \mathrm{~A}$ & $118.8(3)$ \\
\hline $\mathrm{C} 37 \mathrm{~A}-\mathrm{C} 36 \mathrm{~A}-\mathrm{H} 36 \mathrm{~A}$ & 120.6 \\
\hline $\mathrm{C} 35 \mathrm{~A}-\mathrm{C} 36 \mathrm{~A}-\mathrm{H} 36 \mathrm{~A}$ & 120.6 \\
\hline $\mathrm{C} 36 \mathrm{~A}-\mathrm{C} 37 \mathrm{~A}-\mathrm{C} 32 \mathrm{~A}$ & $121.0(3)$ \\
\hline $\mathrm{C} 36 \mathrm{~A}-\mathrm{C} 37 \mathrm{~A}-\mathrm{H} 37 \mathrm{~A}$ & 119.5 \\
\hline $\mathrm{C} 32 \mathrm{~A}-\mathrm{C} 37 \mathrm{~A}-\mathrm{H} 37 \mathrm{~A}$ & 119.5 \\
\hline $\mathrm{O} 31 \mathrm{~A}-\mathrm{N} 31 \mathrm{~A}-\mathrm{O} 32 \mathrm{~A}$ & $123.5(2)$ \\
\hline $\mathrm{O} 31 \mathrm{~A}-\mathrm{N} 31 \mathrm{~A}-\mathrm{C} 35 \mathrm{~A}$ & $118.2(2)$ \\
\hline $\mathrm{O} 32 \mathrm{~A}-\mathrm{N} 31 \mathrm{~A}-\mathrm{C} 35 \mathrm{~A}$ & $118.3(2)$ \\
\hline $\mathrm{C} 5 \mathrm{~A}-\mathrm{C} 4 \mathrm{~A}-\mathrm{N} 3 \mathrm{~A}$ & $103.4(2)$ \\
\hline $\mathrm{C} 5 \mathrm{~A}-\mathrm{C} 4 \mathrm{~A}-\mathrm{C} 41 \mathrm{~A}$ & $130.2(3)$ \\
\hline $\mathrm{N} 3 \mathrm{~A}-\mathrm{C} 4 \mathrm{~A}-\mathrm{C} 41 \mathrm{~A}$ & $126.4(2)$ \\
\hline $\mathrm{O} 41 \mathrm{~A}-\mathrm{C} 41 \mathrm{~A}-\mathrm{C} 4 \mathrm{~A}$ & $126.4(3)$ \\
\hline $\mathrm{O} 41 \mathrm{~A}-\mathrm{C} 41 \mathrm{~A}-\mathrm{H} 41 \mathrm{~A}$ & 116.8 \\
\hline $\mathrm{C} 4 \mathrm{~A}-\mathrm{C} 41 \mathrm{~A}-\mathrm{H} 41 \mathrm{~A}$ & 116.8 \\
\hline $\mathrm{N} 1 \mathrm{~A}-\mathrm{C} 5 \mathrm{~A}-\mathrm{C} 4 \mathrm{~A}$ & $112.9(2)$ \\
\hline $\mathrm{N} 1 \mathrm{~A}-\mathrm{C} 5 \mathrm{~A}-\mathrm{Cl} 5 \mathrm{~A}$ & $120.2(2)$ \\
\hline $\mathrm{C} 5 \mathrm{~A}-\mathrm{N} 1 \mathrm{~A}-\mathrm{C} 2 \mathrm{~A}-\mathrm{N} 3 \mathrm{~A}$ & $0.0(3)$ \\
\hline $\mathrm{C} 5 \mathrm{~A}-\mathrm{N} 1 \mathrm{~A}-\mathrm{C} 2 \mathrm{~A}-\mathrm{C} 21 \mathrm{~A}$ & $-178.5(3)$ \\
\hline $\mathrm{N} 1 \mathrm{~A}-\mathrm{C} 2 \mathrm{~A}-\mathrm{C} 21 \mathrm{~A}-\mathrm{C} 22 \mathrm{~A}$ & $-106.8(3)$ \\
\hline $\mathrm{N} 3 \mathrm{~A}-\mathrm{C} 2 \mathrm{~A}-\mathrm{C} 21 \mathrm{~A}-\mathrm{C} 22 \mathrm{~A}$ & $74.9(4)$ \\
\hline $\mathrm{C} 2 \mathrm{~A}-\mathrm{C} 21 \mathrm{~A}-\mathrm{C} 22 \mathrm{~A}-\mathrm{C} 23 \mathrm{~A}$ & $175.1(3)$ \\
\hline $\mathrm{C} 21 \mathrm{~A}-\mathrm{C} 22 \mathrm{~A}-\mathrm{C} 23 \mathrm{~A}-\mathrm{C} 24 \mathrm{~A}$ & $179.0(3)$ \\
\hline $\mathrm{N} 1 \mathrm{~A}-\mathrm{C} 2 \mathrm{~A}-\mathrm{N} 3 \mathrm{~A}-\mathrm{C} 4 \mathrm{~A}$ & $0.0(3)$ \\
\hline $\mathrm{C} 21 \mathrm{~A}-\mathrm{C} 2 \mathrm{~A}-\mathrm{N} 3 \mathrm{~A}-\mathrm{C} 4 \mathrm{~A}$ & $178.5(3)$ \\
\hline $\mathrm{N} 1 \mathrm{~A}-\mathrm{C} 2 \mathrm{~A}-\mathrm{N} 3 \mathrm{~A}-\mathrm{C} 31 \mathrm{~A}$ & $176.8(2)$ \\
\hline $\mathrm{C} 21 \mathrm{~A}-\mathrm{C} 2 \mathrm{~A}-\mathrm{N} 3 \mathrm{~A}-\mathrm{C} 31 \mathrm{~A}$ & $-4.7(4)$ \\
\hline $\mathrm{C} 2 \mathrm{~A}-\mathrm{N} 3 \mathrm{~A}-\mathrm{C} 31 \mathrm{~A}-\mathrm{C} 32 \mathrm{~A}$ & $-104.2(3)$ \\
\hline $\mathrm{C} 4 \mathrm{~A}-\mathrm{N} 3 \mathrm{~A}-\mathrm{C} 31 \mathrm{~A}-\mathrm{C} 32 \mathrm{~A}$ & $71.9(3)$ \\
\hline $\mathrm{N} 3 \mathrm{~A}-\mathrm{C} 31 \mathrm{~A}-\mathrm{C} 32 \mathrm{~A}-\mathrm{C} 37 \mathrm{~A}$ & $12.6(4)$ \\
\hline $\mathrm{N} 3 \mathrm{~A}-\mathrm{C} 31 \mathrm{~A}-\mathrm{C} 32 \mathrm{~A}-\mathrm{C} 33 \mathrm{~A}$ & $-166.4(2)$ \\
\hline $\mathrm{C} 37 \mathrm{~A}-\mathrm{C} 32 \mathrm{~A}-\mathrm{C} 33 \mathrm{~A}-\mathrm{C} 34 \mathrm{~A}$ & $-2.0(4)$ \\
\hline $\mathrm{C} 31 \mathrm{~A}-\mathrm{C} 32 \mathrm{~A}-\mathrm{C} 33 \mathrm{~A}-\mathrm{C} 34 \mathrm{~A}$ & $177.0(2)$ \\
\hline
\end{tabular}

\begin{tabular}{|c|c|}
\hline $\mathrm{C} 5 \mathrm{~B}-\mathrm{C} 4 \mathrm{~B}-\mathrm{N} 3 \mathrm{~B}$ & $104.0(2)$ \\
\hline $\mathrm{C} 5 \mathrm{~B}-\mathrm{C} 4 \mathrm{~B}-\mathrm{C} 41 \mathrm{~B}$ & $128.8(3)$ \\
\hline $\mathrm{N} 3 \mathrm{~B}-\mathrm{C} 4 \mathrm{~B}-\mathrm{C} 41 \mathrm{~B}$ & $127.2(2)$ \\
\hline $\mathrm{O} 41 \mathrm{~B}-\mathrm{C} 41 \mathrm{~B}-\mathrm{C} 4 \mathrm{~B}$ & $126.3(3)$ \\
\hline $\mathrm{O} 41 \mathrm{~B}-\mathrm{C} 41 \mathrm{~B}-\mathrm{H} 41 \mathrm{~B}$ & 116.8 \\
\hline $\mathrm{C} 4 \mathrm{~B}-\mathrm{C} 41 \mathrm{~B}-\mathrm{H} 41 \mathrm{~B}$ & 116.8 \\
\hline $\mathrm{N} 1 \mathrm{~B}-\mathrm{C} 5 \mathrm{~B}-\mathrm{C} 4 \mathrm{~B}$ & $112.6(2)$ \\
\hline $\mathrm{N} 1 \mathrm{~B}-\mathrm{C} 5 \mathrm{~B}-\mathrm{Cl} 5 \mathrm{~B}$ & $121.4(2)$ \\
\hline $\mathrm{C} 4 \mathrm{~B}-\mathrm{C} 5 \mathrm{~B}-\mathrm{Cl} 5 \mathrm{~B}$ & $126.0(2)$ \\
\hline $\mathrm{C} 2 \mathrm{~B}-\mathrm{C} 21 \mathrm{~B}-\mathrm{C} 22 \mathrm{~B}$ & $113.8(3)$ \\
\hline $\mathrm{C} 2 \mathrm{~B}-\mathrm{C} 21 \mathrm{~B}-\mathrm{H} 21 \mathrm{~B}$ & 108.8 \\
\hline $\mathrm{C} 22 \mathrm{~B}-\mathrm{C} 21 \mathrm{~B}-\mathrm{H} 21 \mathrm{~B}$ & 108.8 \\
\hline $\mathrm{C} 2 \mathrm{~B}-\mathrm{C} 21 \mathrm{~B}-\mathrm{H} 22 \mathrm{~B}$ & 108.8 \\
\hline $\mathrm{C} 22 \mathrm{~B}-\mathrm{C} 21 \mathrm{~B}-\mathrm{H} 22 \mathrm{~B}$ & 108.8 \\
\hline $\mathrm{H} 21 \mathrm{~B}-\mathrm{C} 21 \mathrm{~B}-\mathrm{H} 22 \mathrm{~B}$ & 107.7 \\
\hline $\mathrm{C} 23 \mathrm{~B}-\mathrm{C} 22 \mathrm{~B}-\mathrm{C} 21 \mathrm{~B}$ & $120.0(4)$ \\
\hline $\mathrm{C} 23 \mathrm{~B}-\mathrm{C} 22 \mathrm{~B}-\mathrm{H} 23 \mathrm{~B}$ & 107.3 \\
\hline $\mathrm{C} 21 \mathrm{~B}-\mathrm{C} 22 \mathrm{~B}-\mathrm{H} 23 \mathrm{~B}$ & 107.3 \\
\hline $\mathrm{C} 23 \mathrm{~B}-\mathrm{C} 22 \mathrm{~B}-\mathrm{H} 24 \mathrm{~B}$ & 107.3 \\
\hline $\mathrm{C} 21 \mathrm{~B}-\mathrm{C} 22 \mathrm{~B}-\mathrm{H} 24 \mathrm{~B}$ & 107.3 \\
\hline $\mathrm{H} 23 \mathrm{~B}-\mathrm{C} 22 \mathrm{~B}-\mathrm{H} 24 \mathrm{~B}$ & 106.9 \\
\hline $\mathrm{C} 22 \mathrm{~B}-\mathrm{C} 23 \mathrm{~B}-\mathrm{C} 24 \mathrm{~B}$ & $112.5(6)$ \\
\hline $\mathrm{C} 22 \mathrm{~B}-\mathrm{C} 23 \mathrm{~B}-\mathrm{H} 25 \mathrm{~B}$ & 109.1 \\
\hline $\mathrm{C} 24 \mathrm{~B}-\mathrm{C} 23 \mathrm{~B}-\mathrm{H} 25 \mathrm{~B}$ & 109.1 \\
\hline $\mathrm{C} 22 \mathrm{~B}-\mathrm{C} 23 \mathrm{~B}-\mathrm{H} 26 \mathrm{~B}$ & 109.1 \\
\hline $\mathrm{C} 24 \mathrm{~B}-\mathrm{C} 23 \mathrm{~B}-\mathrm{H} 26 \mathrm{~B}$ & 109.1 \\
\hline $\mathrm{H} 25 \mathrm{~B}-\mathrm{C} 23 \mathrm{~B}-\mathrm{H} 26 \mathrm{~B}$ & 107.8 \\
\hline $\mathrm{H} 23 \mathrm{C}-\mathrm{C} 22 \mathrm{C}-\mathrm{H} 24 \mathrm{C}$ & 106.4 \\
\hline $\mathrm{H} 27 \mathrm{C}-\mathrm{C} 24 \mathrm{C}-\mathrm{H} 28 \mathrm{C}$ & 109.5 \\
\hline $\mathrm{H} 27 \mathrm{C}-\mathrm{C} 24 \mathrm{C}-\mathrm{H} 29 \mathrm{C}$ & 109.5 \\
\hline $\mathrm{H} 28 \mathrm{C}-\mathrm{C} 24 \mathrm{C}-\mathrm{H} 29 \mathrm{C}$ & 109.5 \\
\hline $\mathrm{C} 5 \mathrm{~B}-\mathrm{N} 1 \mathrm{~B}-\mathrm{C} 2 \mathrm{~B}-\mathrm{N} 3 \mathrm{~B}$ & $0.0(3)$ \\
\hline $\mathrm{C} 5 \mathrm{~B}-\mathrm{N} 1 \mathrm{~B}-\mathrm{C} 2 \mathrm{~B}-\mathrm{C} 21 \mathrm{~B}$ & $176.7(3)$ \\
\hline $\mathrm{N} 1 \mathrm{~B}-\mathrm{C} 2 \mathrm{~B}-\mathrm{N} 3 \mathrm{~B}-\mathrm{C} 4 \mathrm{~B}$ & $0.0(3)$ \\
\hline $\mathrm{C} 21 \mathrm{~B}-\mathrm{C} 2 \mathrm{~B}-\mathrm{N} 3 \mathrm{~B}-\mathrm{C} 4 \mathrm{~B}$ & $-176.8(3)$ \\
\hline $\mathrm{N} 1 \mathrm{~B}-\mathrm{C} 2 \mathrm{~B}-\mathrm{N} 3 \mathrm{~B}-\mathrm{C} 31 \mathrm{~B}$ & $179.6(2)$ \\
\hline $\mathrm{C} 21 \mathrm{~B}-\mathrm{C} 2 \mathrm{~B}-\mathrm{N} 3 \mathrm{~B}-\mathrm{C} 31 \mathrm{~B}$ & $2.8(4)$ \\
\hline $\mathrm{C} 2 \mathrm{~B}-\mathrm{N} 3 \mathrm{~B}-\mathrm{C} 31 \mathrm{~B}-\mathrm{C} 32 \mathrm{~B}$ & $97.8(3)$ \\
\hline $\mathrm{C} 4 \mathrm{~B}-\mathrm{N} 3 \mathrm{~B}-\mathrm{C} 31 \mathrm{~B}-\mathrm{C} 32 \mathrm{~B}$ & $-82.7(3)$ \\
\hline $\mathrm{N} 3 \mathrm{~B}-\mathrm{C} 31 \mathrm{~B}-\mathrm{C} 32 \mathrm{~B}-\mathrm{C} 37 \mathrm{~B}$ & $12.5(4)$ \\
\hline $\mathrm{N} 3 \mathrm{~B}-\mathrm{C} 31 \mathrm{~B}-\mathrm{C} 32 \mathrm{~B}-\mathrm{C} 33 \mathrm{~B}$ & $-170.0(2)$ \\
\hline $\mathrm{C} 37 \mathrm{~B}-\mathrm{C} 32 \mathrm{~B}-\mathrm{C} 33 \mathrm{~B}-\mathrm{C} 34 \mathrm{~B}$ & $-0.7(4)$ \\
\hline $\mathrm{C} 31 \mathrm{~B}-\mathrm{C} 32 \mathrm{~B}-\mathrm{C} 33 \mathrm{~B}-\mathrm{C} 34 \mathrm{~B}$ & $-178.3(3)$ \\
\hline $\mathrm{C} 32 \mathrm{~B}-\mathrm{C} 33 \mathrm{~B}-\mathrm{C} 34 \mathrm{~B}-\mathrm{C} 35 \mathrm{~B}$ & $0.5(4)$ \\
\hline $\mathrm{C} 33 \mathrm{~B}-\mathrm{C} 34 \mathrm{~B}-\mathrm{C} 35 \mathrm{~B}-\mathrm{C} 36 \mathrm{~B}$ & $0.2(4)$ \\
\hline $\mathrm{C} 33 \mathrm{~B}-\mathrm{C} 34 \mathrm{~B}-\mathrm{C} 35 \mathrm{~B}-\mathrm{N} 31 \mathrm{~B}$ & $179.4(3)$ \\
\hline $\mathrm{C} 34 \mathrm{~B}-\mathrm{C} 35 \mathrm{~B}-\mathrm{C} 36 \mathrm{~B}-\mathrm{C} 37 \mathrm{~B}$ & $-0.7(4)$ \\
\hline
\end{tabular}




$\begin{array}{ll}\mathrm{C} 32 \mathrm{~A}-\mathrm{C} 33 \mathrm{~A}-\mathrm{C} 34 \mathrm{~A}-\mathrm{C} 35 \mathrm{~A} & 0.4(4) \\ \mathrm{C} 33 \mathrm{~A}-\mathrm{C} 34 \mathrm{~A}-\mathrm{C} 35 \mathrm{~A}-\mathrm{C} 36 \mathrm{~A} & 1.8(4) \\ \mathrm{C} 33 \mathrm{~A}-\mathrm{C} 34 \mathrm{~A}-\mathrm{C} 35 \mathrm{~A}-\mathrm{N} 31 \mathrm{~A} & -178.2(2) \\ \mathrm{C} 34 \mathrm{~A}-\mathrm{C} 35 \mathrm{~A}-\mathrm{C} 36 \mathrm{~A}-\mathrm{C} 37 \mathrm{~A} & -2.4(4) \\ \mathrm{N} 31 \mathrm{~A}-\mathrm{C} 35 \mathrm{~A}-\mathrm{C} 36 \mathrm{~A}-\mathrm{C} 37 \mathrm{~A} & 177.6(2) \\ \mathrm{C} 35 \mathrm{~A}-\mathrm{C} 36 \mathrm{~A}-\mathrm{C} 37 \mathrm{~A}-\mathrm{C} 32 \mathrm{~A} & 0.7(4) \\ \mathrm{C} 33 \mathrm{~A}-\mathrm{C} 32 \mathrm{~A}-\mathrm{C} 37 \mathrm{~A}-\mathrm{C} 36 \mathrm{~A} & 1.4(4) \\ \mathrm{C} 31 \mathrm{~A}-\mathrm{C} 32 \mathrm{~A}-\mathrm{C} 37 \mathrm{~A}-\mathrm{C} 36 \mathrm{~A} & -177.6(3) \\ \mathrm{C} 36 \mathrm{~A}-\mathrm{C} 35 \mathrm{~A}-\mathrm{N} 31 \mathrm{~A}-\mathrm{O} 31 \mathrm{~A} & -177.8(2) \\ \mathrm{C} 34 \mathrm{~A}-\mathrm{C} 35 \mathrm{~A}-\mathrm{N} 31 \mathrm{~A}-\mathrm{O} 31 \mathrm{~A} & 2.2(4) \\ \mathrm{C} 36 \mathrm{~A}-\mathrm{C} 35 \mathrm{~A}-\mathrm{N} 31 \mathrm{~A}-\mathrm{O} 32 \mathrm{~A} & 2.1(4) \\ \mathrm{C} 34 \mathrm{~A}-\mathrm{C} 35 \mathrm{~A}-\mathrm{N} 31 \mathrm{~A}-\mathrm{O} 32 \mathrm{~A} & -178.0(2) \\ \mathrm{C} 2 \mathrm{~A}-\mathrm{N} 3 \mathrm{~A}-\mathrm{C} 4 \mathrm{~A}-\mathrm{C} 5 \mathrm{~A} & -0.1(3) \\ \mathrm{C} 31 \mathrm{~A}-\mathrm{N} 3 \mathrm{~A}-\mathrm{C} 4 \mathrm{~A}-\mathrm{C} 5 \mathrm{~A} & -176.9(2) \\ \mathrm{C} 2 \mathrm{~A}-\mathrm{N} 3 \mathrm{~A}-\mathrm{C} 4 \mathrm{~A}-\mathrm{C} 41 \mathrm{~A} & 179.7(3) \\ \mathrm{C} 31 \mathrm{~A}-\mathrm{N} 3 \mathrm{~A}-\mathrm{C} 4 \mathrm{~A}-\mathrm{C} 41 \mathrm{~A} & 2.9(4) \\ \mathrm{C} 5 \mathrm{~A}-\mathrm{C} 4 \mathrm{~A}-\mathrm{C} 41 \mathrm{~A}-\mathrm{O} 41 \mathrm{~A} & -178.4(3) \\ \mathrm{N} 3 \mathrm{~A}-\mathrm{C} 4 \mathrm{~A}-\mathrm{C} 41 \mathrm{~A}-\mathrm{O} 41 \mathrm{~A} & 1.8(5) \\ \mathrm{C} 2 \mathrm{~A}-\mathrm{N} 1 \mathrm{~A}-\mathrm{C} 5 \mathrm{~A}-\mathrm{C} 4 \mathrm{~A} & -0.1(3) \\ \text { C2A-N1A-C5A-C15A } & 179.7(2) \\ \text { N3A-C4A-C5A-N1A } & 0.1(3) \\ \text { C41A-C4A-C5A-N1A } & -179.7(3) \\ \text { N3A-C4A-C5A-C15A } & -179.6(2) \\ \text { C41A-C4A-C5A-C15A } & 0.6(5)\end{array}$

$\begin{array}{ll}\mathrm{N} 31 \mathrm{~B}-\mathrm{C} 35 \mathrm{~B}-\mathrm{C} 36 \mathrm{~B}-\mathrm{C} 37 \mathrm{~B} & -180.0(3) \\ \mathrm{C} 33 \mathrm{~B}-\mathrm{C} 32 \mathrm{~B}-\mathrm{C} 37 \mathrm{~B}-\mathrm{C} 36 \mathrm{~B} & 0.1(4) \\ \mathrm{C} 31 \mathrm{~B}-\mathrm{C} 32 \mathrm{~B}-\mathrm{C} 37 \mathrm{~B}-\mathrm{C} 36 \mathrm{~B} & 177.6(3) \\ \mathrm{C} 35 \mathrm{~B}-\mathrm{C} 36 \mathrm{~B}-\mathrm{C} 37 \mathrm{~B}-\mathrm{C} 32 \mathrm{~B} & 0.5(4) \\ \mathrm{C} 36 \mathrm{~B}-\mathrm{C} 35 \mathrm{~B}-\mathrm{N} 31 \mathrm{~B}-\mathrm{O} 31 \mathrm{~B} & -177.7(3) \\ \mathrm{C} 34 \mathrm{~B}-\mathrm{C} 35 \mathrm{~B}-\mathrm{N} 31 \mathrm{~B}-\mathrm{O} 31 \mathrm{~B} & 3.0(4) \\ \mathrm{C} 36 \mathrm{~B}-\mathrm{C} 35 \mathrm{~B}-\mathrm{N} 31 \mathrm{~B}-\mathrm{O} 32 \mathrm{~B} & 2.8(4) \\ \mathrm{C} 34 \mathrm{~B}-\mathrm{C} 35 \mathrm{~B}-\mathrm{N} 31 \mathrm{~B}-\mathrm{O} 32 \mathrm{~B} & -176.5(3) \\ \mathrm{C} 2 \mathrm{~B}-\mathrm{N} 3 \mathrm{~B}-\mathrm{C} 4 \mathrm{~B}-\mathrm{C} 5 \mathrm{~B} & 0.0(3) \\ \mathrm{C} 31 \mathrm{~B}-\mathrm{N} 3 \mathrm{~B}-\mathrm{C} 4 \mathrm{~B}-\mathrm{C} 5 \mathrm{~B} & -179.6(2) \\ \mathrm{C} 2 \mathrm{~B}-\mathrm{N} 3 \mathrm{~B}-\mathrm{C} 4 \mathrm{~B}-\mathrm{C} 41 \mathrm{~B} & 180.0(3) \\ \mathrm{C} 31 \mathrm{~B}-\mathrm{N} 3 \mathrm{~B}-\mathrm{C} 4 \mathrm{~B}-\mathrm{C} 41 \mathrm{~B} & 0.4(4) \\ \mathrm{C} 5 \mathrm{~B}-\mathrm{C} 4 \mathrm{~B}-\mathrm{C} 41 \mathrm{~B}-\mathrm{O} 41 \mathrm{~B} & 179.0(3) \\ \mathrm{N} 3 \mathrm{~B}-\mathrm{C} 4 \mathrm{~B}-\mathrm{C} 41 \mathrm{~B}-\mathrm{O} 41 \mathrm{~B} & -0.9(5) \\ \mathrm{C} 2 \mathrm{~B}-\mathrm{N} 1 \mathrm{~B}-\mathrm{C} 5 \mathrm{~B}-\mathrm{C} 4 \mathrm{~B} & 0.0(3) \\ \mathrm{C} 2 \mathrm{~B}-\mathrm{N} 1 \mathrm{~B}-\mathrm{C} 5 \mathrm{~B}-\mathrm{Cl} 5 \mathrm{~B} & -179.8(2) \\ \mathrm{N} 3 \mathrm{~B}-\mathrm{C} 4 \mathrm{~B}-\mathrm{C} 5 \mathrm{~B}-\mathrm{N} 1 \mathrm{~B} & 0.0(3) \\ \mathrm{C} 41 \mathrm{~B}-\mathrm{C} 4 \mathrm{~B}-\mathrm{C} 5 \mathrm{~B}-\mathrm{N} 1 \mathrm{~B} & 180.0(3) \\ \mathrm{N} 3 \mathrm{~B}-\mathrm{C} 4 \mathrm{~B}-\mathrm{C} 5 \mathrm{~B}-\mathrm{C} 15 \mathrm{~B} & 179.8(2) \\ \mathrm{C} 41 \mathrm{~B}-\mathrm{C} 4 \mathrm{~B}-\mathrm{C} 5 \mathrm{~B}-\mathrm{Cl} 5 \mathrm{~B} & -0.2(4) \\ \mathrm{N} 1 \mathrm{~B}-\mathrm{C} 2 \mathrm{~B}-\mathrm{C} 21 \mathrm{~B}-\mathrm{C} 22 \mathrm{~B} & 16.0(5) \\ \mathrm{N} 3 \mathrm{~B}-\mathrm{C} 2 \mathrm{~B}-\mathrm{C} 21 \mathrm{~B}-\mathrm{C} 22 \mathrm{~B} & -167.5(3) \\ \mathrm{C} 2 \mathrm{~B}-\mathrm{C} 21 \mathrm{~B}-\mathrm{C} 22 \mathrm{~B}-\mathrm{C} 23 \mathrm{~B} & 70.9(6) \\ \mathrm{C} 21 \mathrm{~B}-\mathrm{C} 22 \mathrm{~B}-\mathrm{C} 23 \mathrm{~B}-\mathrm{C} 24 \mathrm{~B} & -173.9(5) \\ \end{array}$

